\title{
MicroRNA-1291 mediates cell proliferation and tumorigenesis by downregulating MED1 in prostate cancer
}

\author{
QI CAI $^{1}$, AN ZHAO $^{2}$, LIGANG REN $^{1}$, JING CHEN $^{1}$, KAISEN LIAO $^{1}$, ZHANSHI WANG $^{1}$ and WEI ZHANG ${ }^{1}$ \\ ${ }^{1}$ Department of Urology, Tongde Hospital of Zhejiang Province, Hangzhou, Zhejiang 310012; \\ ${ }^{2}$ Zhejiang Cancer Research Institute, Zhejiang Cancer Hospital, Hangzhou, Zhejiang 310022, P.R. China
}

Received May 28, 2018; Accepted January 15, 2019

DOI: 10.3892/ol.2019.9980

\begin{abstract}
RNAs are important factors involved in the regulation of tumor development. miR-1291 was found to have regulatory effects in many tumors, but its role in prostate cancer (PCa) still remains unclear. We explored the expression of miR-1291 in $\mathrm{PCa}$ to reveal its role in regulating the progression of $\mathrm{PCa}$ as well as its underlying mechanism. Reverse transcription-quantitative polymerase chain reaction (RT-qPCR) was used to detect the expression of miR-1291 in PCa tissues and cell lines compared to normal tissues and cell lines. miR-1291 mimics and inhibitors were applied to overexpress or inhibit the level of miR-1291 in PCa cells. The ability of cell proliferation was measured using MTT assay, and cell cycle distribution was determined by flow cytometry. The potential target of miR-1291 was identified via western blot analysis and luciferase assays. Then a xenograft model was established to explore the function of miR-1291 in PCa in vivo. The results revealed that the expression level of miR-1291 was significantly lower in the PCa tissues than that in the normal adjacent tissues. In PCa-derived cells, there was also a downregulated expression level of miR-1291. Overexpression of miR-1291 obviously inhibited DU-145 cell proliferation and induced cell cycle transition from $\mathrm{G} 0 / \mathrm{G} 1$ to $\mathrm{S}$ phase. However, inhibition of miR-1291 promoted the growth of LNCaP cells, and promoted the cell cycle transition to $\mathrm{S}$ phase and G2/M phase. MED1 was proven to be a potential target gene of miR-1291, and miR-1291 significantly inhibited its expression. At the in vivo level, overexpression of miR-1291 inhibited the growth of xenograft tumors and significantly inhibited the expression of MED1 protein. Our study demonstrated that miR-1291 inhibits cell proliferation and tumorigenesis of PCa via MED1, which might provide a novel target for $\mathrm{PCa}$ diagnosis and biological therapy.
\end{abstract}

Correspondence to: Dr Wei Zhang, Department of Urology, Tongde Hospital of Zhejiang Province, 234 Gucui Road, Xihu, Hangzhou, Zhejiang 310012, P.R. China

E-mail:mkcde016@163.com

Key words: miR-1291, proliferation, tumorigenesis, MED1, prostate cancer

\section{Introduction}

According to the latest cancer data, prostate cancer (PCa) has the highest incidence of cancers among male tumors worldwide. New cases in the United States in 2017 accounted for $19 \%$ of total malignancies (1). Although the treatment of PCa has achieved curative effect, and the 5-year survival rate of $\mathrm{PCa}$ is relatively optimistic compared to other malignant tumors (2-4), there are still many problems that need to be solved in the diagnosis and treatment of PCa. Finding more effective biological diagnostic and therapeutic targets remains a hot topic in PCa research $(5,6)$.

MicroRNAs are a type of specific RNA molecules of 20-22 nucleotides in length that can inhibit the expression of target genes through specific binding to the 3'-untranslated region (3'-UTR) of their target genes, thereby exerting their role in regulating various molecular biological processes $(7,8)$. In tumors, miRNAs affect the biological behavior in many aspects such as tumor occurrence, metastasis, invasion, microenvironment, and autophagy (9-11). Several miRNAs have been identified to be involved in the development and progression of PCa. miR-34a could inhibit PCa stem cells and invasion directly through repressing the expression of CD44, miR-195 targets RPS6KB1, inhibits PCa proliferation and metastasis, and also, miR-409-3p/-5p promotes the development, and metastasis to bone via epithelial-to-mesenchymal transition (12-14). In addition, miR-940 has been shown to suppress PCa cell invasion and migration via inhibiting the expression of MIEN1 (15).

miR-1291 has been reported to be involved in the regulation of multiple cancers. In different types of cancers, it could regulate the growth and metastasis of tumor cells by regulating different specific target genes. For example, in pancreatic cancer, it suppresses tumorigenesis and cell growth via targeting the FOXA2-AGR2 axis; in esophageal squamous cell cancer, it inhibits cell proliferation and invasion through mucin 1 and accelerates cell apoptosis; in renal cell carcinoma, it functions as a tumor suppressor by regulating glucose transporter 1 (16-18). Also, together with miR-133, miR-1291 acts as angio-miR to modulate HUVEC angiogenesis (19). However, in $\mathrm{PCa}$, the expression and role of miR-1291 have not been studied yet.

In this study, we first detected the expression level of miR-1291 in surgically removed PCa tissues compared to 
adjacent normal tissues. Also, the expression of miR-1291 in PCa cell lines was measured. With 3-(4,5-dimethylthiazol-2-yl)-2,5-diphenyltetrazolium bromide (MTT) assay and cell cycle detection, we demonstrated that miR-1291 could affect the cell proliferation of PCa cells. Moreover, MED1 was identified as a direct target for miR-1291 in PCa. Taken together, miR-1291 was found to act as a tumor suppressor in PCa via MED1 and has tpotential to be a diagnostic and therapeutic target.

\section{Materials and methods}

Patients and tissue samples. All 98 paired PCa tissues and adjacent normal tissues were obtained from male patients (72.1 \pm 6.3 years of age) who underwent surgical treatment in Tongde Hospital of Zhejiang Province (Hangzhou, China) between June 2014 and August 2017. None of the patients received preoperative radiotherapy or chemotherapy. All samples were immediately stored in liquid nitrogen after excision. The experiments were approved by the Ethics Committee of Tongde Hospital of Zhejiang Province and all the patients or the guardians signed an informed consent.

Cell lines and culture. The four cell lines, including three PCa-derived cell lines DU-145 (cat. no. BNCC338240), PC3 (cat. no. BNCC337715), LNCaP (cat. no. BNCC337702) and one normal prostate epithelial cell line RWPE-1 (cat. no. BNCC100292), were obtained from BeNa Culture Collection Co. (Beijing, China; http://www.bnbio.com/). Dulbecco's modified Eagle's medium (DMEM; Thermo Fisher Scientific, Inc., Waltham, MA, USA) containing 10\% fetal bovine serum (FBS) and 1\% penicillin-streptomycin solution (both from Gibco; Thermo Fisher Scientific, Inc., Rockville, MD, USA) was utilized to maintain the cells. The cells were cultured in moist air at $37^{\circ} \mathrm{C}$ containing $5 \% \mathrm{CO}_{2}$.

$R N A$ isolation and reverse transcription-quantitative polymerase chain reaction (RT-qPCR). For $\mathrm{PCa}$ and normal tissues, a total of $1 \mathrm{ml}$ TRIzol solution (Invitrogen; Thermo Fisher Scientific, Inc.) was added for lysis. For experimental cells, $3 \times 10^{5}$ cells were lysed by adding $1 \mathrm{ml}$ TRIzol solution. The total RNA was measured for purity concentration by a UV spectrophotometer (Hitachi, Ltd., Tokyo, Japan) and stored at $-80^{\circ} \mathrm{C}$.

The PrimeScript RT reagent (Takara Bio, Inc., Kusatsu, Japan) was used to perform reverse transcription according to the manufacturer's instructions. The SYBR-Green Master Mix I (Takara Bio, Inc.) was employed to perform the RT-qPCR using the ABI 7500 Fast Real-Time PCR System (Applied Biosystems, Inc.; Thermo Fisher Scientific, Inc., Foster City, CA, USA). U6 was applied as internal control for all miRNA samples and GAPDH for all mRNA samples. The reaction steps were as follows: pre-denaturation for $30 \mathrm{sec}$ at $95^{\circ} \mathrm{C}$; followed by 45 cycles of $5 \mathrm{sec}$ at $95^{\circ} \mathrm{C}$ and $30 \mathrm{sec}$ at $60^{\circ} \mathrm{C}$ per cycle, and finally a dissolution medium was added. The relative expression levels were measured using the $2^{-\Delta \Delta \mathrm{Cq}}$ method (20). All primer probes were designed by Guangzhou RiboBio Co., Ltd. (Guangzhou, China). Each experiment was performed in triplicate. The primers sequences used were as follows: MED1 forward, 5'-CCT TTA GAA AGG CAG AAC TCC TCT TCC
GGA TCA CCC CGG-3' and reverse, 5'-CCG GGG TGA TCC GGA AGA GGA GTT CTG CCT TTC TAA AGG-3'; miR-1291 forward, 5'-ACA CTC CAG CTG GGT GGC CCT GAC TGA AGA CC-3' and reverse, 5'-TGG TGT CGT GGA GTC G-3'; GAPDH forward, 5'-TGA CTT CAA CAG CGA CAC CCA-3' and reverse, 5'-GGA GTG TTG GAG AAG TCA TAT TAC-3'; U6 forward, 5'-CTC GCT TCG GCA GCA CAT AT-3' and reverse, 5'-TTG CGT GTC ATC CTT GCG-3'.

Cell transfection of miR-1291 and pcDNA-MEDI. miR-1291 mimics, negative control (NC), inhibitors, inhibitors negative control (INC) and pcDNA-MED1 were designed and synthesized by the Guangzhou RiboBio Co., Ltd. For transfection, appropriate amount of cells was planted in a 6-well plate. When the confluence reached $50-60 \%$, the appropriate amount of miR-1291 mimics, NC, inhibitors, INC or pcDNA-MED1, were added, using Lipofectamine (Invitrogen; Thermo Fisher Scientific, Inc.) according to the manufacturer's instructions. The efficiency of transfection was determined using RT-qPCR.

MTT assay. MTT assay was applied to detect cell proliferation. A total of $3.5 \times 10^{3}$ cells were seeded into 96 -well plates per well after treatment with miR-1291 mimics, NC, inhibitors or INC. At 0, 24, 48 and 72 h, $0.5 \mathrm{mg} / \mathrm{ml}$ MTT buffer (Thermo Fisher Scientific, Inc.) was added per well and cells were cultured for $2 \mathrm{~h}$ in darkness. Next, the absorbance at $490 \mathrm{~nm}$ was detected using a spectrophotometer (Bio-Rad Laboratories, Inc., Hercules, CA, USA). The experiment was repeated 3 times.

Cell cycle detection. Cell cycle distribution was detected using a flow cytometer (FACSCalibur; BD Biosciences, Franklin Lakes, NJ, USA). DU-145 and LNCaP cells after miR-1291 mimics or inhibitor treatment were harvested and washed with phosphate-buffered saline (PBS) buffer. After centrifugation, at $20^{\circ} \mathrm{C}$ for $5 \mathrm{~min}$ at $1,500 \mathrm{x} \mathrm{g}$, cells were re-suspended in $500 \mu 1$ binding buffer containing $1 \%$ propidium iodide (PI; Vazyme, Nanjing, China). Human TruStain FcX ${ }^{\mathrm{TM}}$ (cat. no. 422301; BioLegend, Inc., San Diego, CA, USA), used as the blocking solution, was added to the cells for incubation at room temperature for $5 \mathrm{~min}$. Then, the cell cycle distribution was measured and recorded. Data were analyzed using the CellQuest Pro software (version 3.3; BD Biosciences).

Luciferase assay. The constructed 3'-UTR sequence containing wild-type or mutated miR-1291 binding sequence of MED1 was inserted into the pLG3 promoter vector (Promega Corpo., Madison, WI, USA), respectively (pLG3-MED1-WT or pLG3-MED1-Mutant). DU-145 cells were seeded in 6-well plates and transfected with pLG3-MED1-WT or pLG3-MED1-Mutant, miR-1291 mimics and NC using Lipofectamine 3000 (Invitrogen; Thermo Fisher Scientific, Inc.). After $48 \mathrm{~h}$, the relative luciferase activity was measured using the Luciferase assay kit (Promega Corp.).

Western blot analysis. Protein was extracted using the RIPA solution containing $0.5 \mathrm{M}$ EDTA, protease inhibitors and phosphatase inhibitors (both from Beyotime Institute of Biotechnology, Shanghai, China). Then, the protein sample was mixed with SDS-PAGE protein loading buffer (Beyotime Institute of Biotechnology) in a ratio of 1:4. The sample was then 


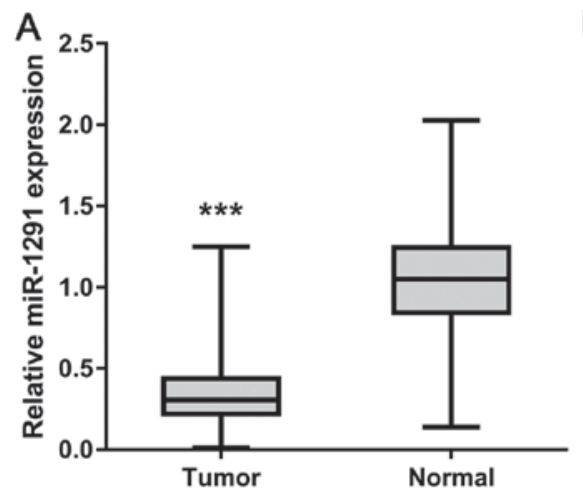

B

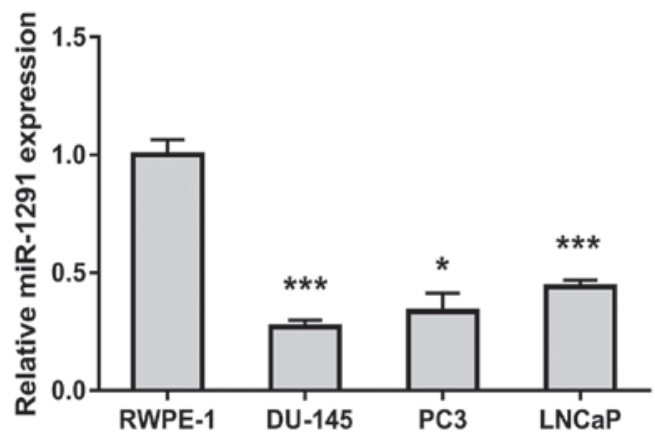

Figure 1. miR-1291 expression in PCa tissues and cell lines. (A) The expression levels of miR-1291 in PCa tissues as well as in the paired adjacent prostate tissues were analyzed by RT-qPCR. (B) miR-1291 expression in RWPE-1 cells and PCa cell lines (DU-145, PC3, LNCaP). Data are shown as mean \pm SD of three separate experiments. ${ }^{*} \mathrm{P}<0.05$ and ${ }^{* * *} \mathrm{P}<0.001$. $\mathrm{PCa}$, prostate cancer.

placed in boiling water for $5 \mathrm{~min}$. A total of $30 \mu \mathrm{g}$ of protein were loaded per lane for the electrophoresis. Proteins were separated by $10 \%$ SDS-PAGE and transferred to polyvinylidene fluoride (PVDF) membranes (EMD Millipore, Billerica, MA, USA). After being blocked with 5\% BSA for $2 \mathrm{~h}$, the membranes were incubated at $4{ }^{\circ} \mathrm{C}$ with specific primary antibodies. Rabbit polyclonal MED1 antibody (dilution, 1:1,000; cat. no. ab64965) and rabbit polyclonal GAPDH antibody (dilution, 1:2,000; cat. no. ab37168) were purchased from Abcam (Cambridge, MA, USA). Membranes were then incubated with secondary goat anti-rabbit (HRP) IgG antibody (dilution, 1:2,000; cat. no. ab6721; Abcam) for $2 \mathrm{~h}$ at room temperature and then washed 3 times with TBST (Beyotime Institute of Biotechnology). The secondary antibody was detected with an enhanced chemiluminescence (ECL) system (Pierce Biotechnology, Inc.; Thermo Fisher Scientific, Inc., Rockford, IL, USA). The experiments were performed in triplicate. The gray value was analyzed using ImageJ software (version 1.38; National Institutes of Health, Bethesda, MD, USA).

Xenograft assay. Thirty nude male mice, weighing 18-22 g, were purchased from the Beijing Weitong Lihua Experimental Animal Technology Co., Ltd. (Beijing, China) to be used in the xenograft model. The mice were housed in a temperature controlled room $\left(21 \pm 2^{\circ} \mathrm{C}\right)$, on a 12:12-h light/dark cycle (lights on at 06:00), and had free access to water and food. The experiment was approved by the Ethics Committee of Tongde Hospital of Zhejiang Province. The 5-6 weeks-old mice were injected with $1 \times 10^{6}$ miR-1291 mimics or NC treated DU-145 cells. The cells were re-suspended in $100 \mu \mathrm{l}$ PBS and injected subcutaneously on the flank of the mouse. Every week, the length and width of the xenograft tumors were measured and their volume was calculated using the formula $0.5 \times$ Length $x$ Width $^{2}$. After 5 weeks, mice were sacrificed and the tumors were removed and weighed. Then, the mRNA and protein of xenografts were extracted, and immunohistochemistry (IHC) was used to detect the expression of MED1.

IHC analyses of MEDI. Xenograft tissues were formalin fixed and paraffin embedded, and then, 4-mm sections were mounted on slides. After antigen retrieval and non-specific serum closure, the slides were attained with rabbit polyclonal MED1 antibody (dilution, 1:100; cat. no. ab64965;
Abcam) and then incubated for $2 \mathrm{~h}$ at $37^{\circ} \mathrm{C}$. After incubation with secondary goat anti-rabbit (HRP) IgG antibody (dilution, 1:500; cat. no. ab6721; Abcam) for $1 \mathrm{~h}$ at $37^{\circ} \mathrm{C}$, DAB reagents (Guangzhou RiboBio Co., Ltd.) and hematoxylin were used for color development. The expression of MED1 in xenograft tumor sections was described and photographed using light microscopy (BX-42; Olympus Corp., Tokyo, Japan) with a magnification of $\mathrm{x} 400$.

Statistical analysis. t-test and ANOVA test, followed by the Least Significant Difference post hoc test, were realized by Statistical Product and Service Solutions (SPSS) 18.0 version software (SPSS, Inc., Chicago, IL, USA) and GraphPad prism version 6.0 software (GraphPad Software, Inc., La Jolla, CA, USA) to analyze the differences. Pearson's correlation test was used to investigate the correlation between miR-1291 expression and the MED1 mRNA level. All the results are expressed as mean $\pm \mathrm{SD}$. $\mathrm{P}<0.05$ was considered to indicate a statistically significant difference.

\section{Results}

miR-1291 is downregulated in PCa tissues and cell lines. To study the relationship of miR-1291 and PCa, we detected the expression level of miR-1291 in 98 paired PCa tissues and adjacent normal tissues. As shown in Fig. 1A, the expression level of miR-1291 in PCa tissues was significantly lower than that in adjacent normal prostate tissues. Also, we obtained three PCa-derived cell lines and measured their miR-1291 level compared to the normal prostate epithelial cell line RWPE-1. The expression of miR-1291 in PCa cells was obviously lower than RWPE-1 cells (Fig. 1B). These results indicate that miR-1291 might act as a tumor suppressor in PCa.

Ectopic miR-1291 effect on cell proliferation and cell cycle of $P C a$. To further study the effects of miR-1291 on PCa cells, we established miR-1291 up- and downregulated cells using miR-1291 mimics and inhibitors. DU-145 cells transfected with miR-1291 mimics showed an increased miR-1291 level compared to the NC group (Fig. 2A), while LNCaP cells transfected with miR-1291 inhibitors showed a decreased miR-1291 level compared to the INC group (Fig. 3A). Using MTT assay, we explored the cell proliferation ability and found that 
A

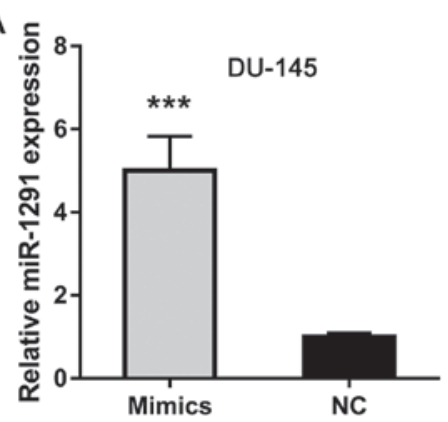

C

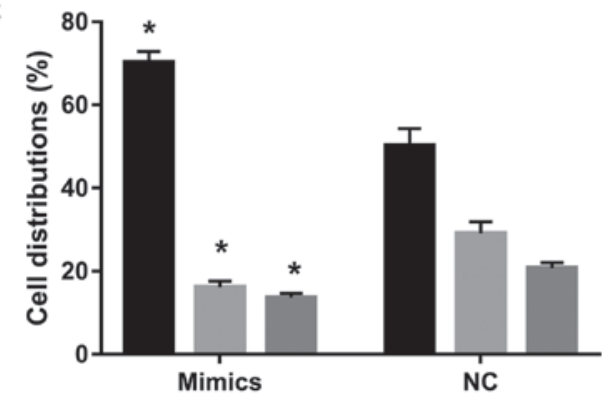

B

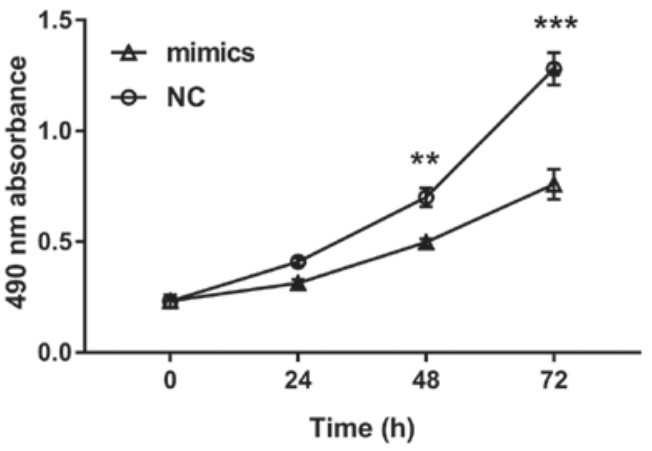

G0/G1

$\mathrm{S}$

G2/M

Figure 2. Overexpression of miR-1291 inhibits proliferation and induced cell cycle of DU-145 cells. (A) DU-145 cells were transfected with miR-1291 mimics or NC. (B) Cell viability was detected by MTT assay. (C) After transfection for 48 h, DU-145 cells were stained with PI, and the distributions of cell cycle were determined using flow cytometric analysis. ${ }^{*} \mathrm{P}<0.05,{ }^{* *} \mathrm{P}<0.01$ and ${ }^{* * * *} \mathrm{P}<0.001$. NC, negative control; PI, propidium iodide.

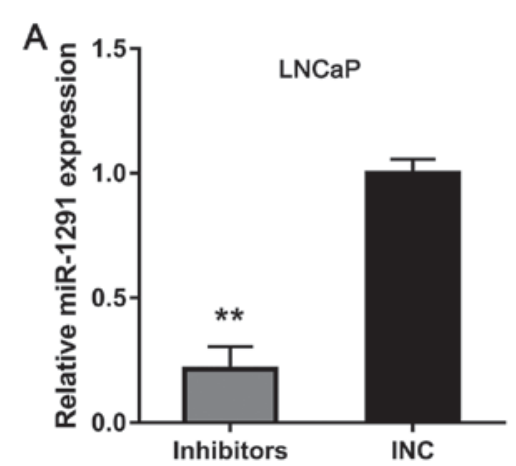

C
B

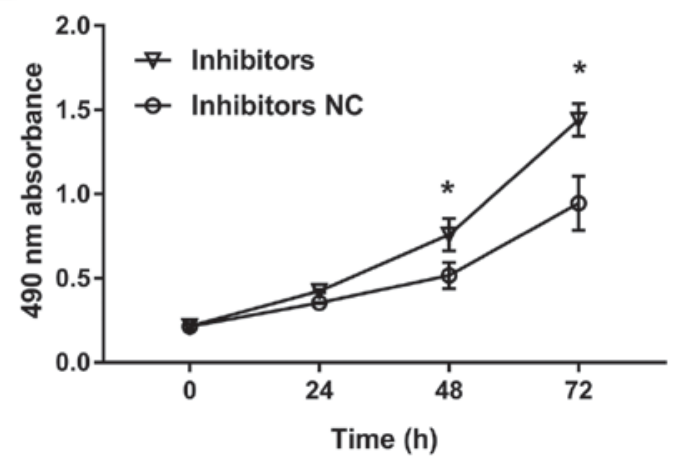

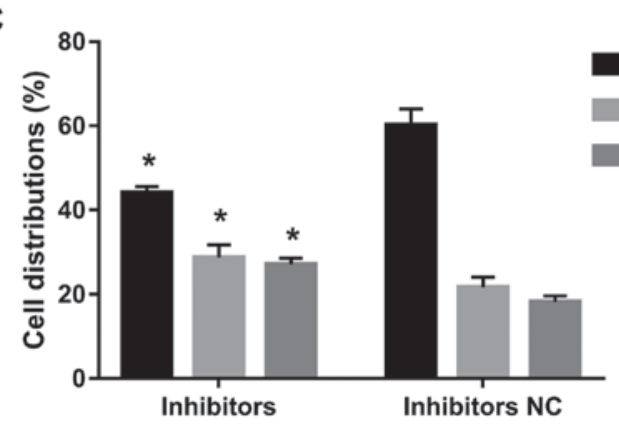

Figure 3. Downregulation of miR-1291 promotes proliferation and cell cycle of LNCaP cells. (A) LNCaP cells were transfected with miR-1291 inhibitors or INC. (B) Cell viability was detected by MTT assay. (C) After transfection for $48 \mathrm{~h}, \mathrm{LNCaP}$ cells were stained with PI, and the distribution of cell cycle was determined using flow cytometric analysis. ${ }^{*} \mathrm{P}<0.05$ and ${ }^{* * *} \mathrm{P}<0.01$. INC, inhibitor negative control; PI, propidium iodide.

overexpression of miR-1291 obviously inhibits cell growth of DU-145 cells (Fig. 2B), but knockdown of miR-1291 promotes the proliferation of LNCaP cells (Fig. 3B). Furthermore, flow cytometry detection revealed that miR-1291 mimics induces cell cycle arrest in G0/G1 phase while miR-1291 inhibitors promote cell cycle transition from G0/G1 phase to $\mathrm{S}$ and
G2/M phase. These data indicate that miR-1291 inhibits cell proliferation and induces cell cycle arrest of PCa cells.

MED1 is a direct target of miR-1291 in PCa. To explain the underlying mechanism of miR-1291 in PCa, due to miRNAs and whether they play their roles via binding to the 
A

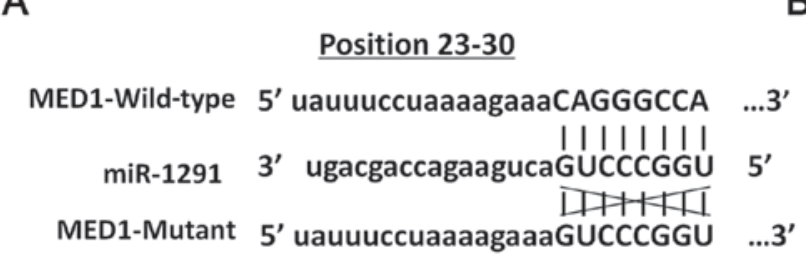

B

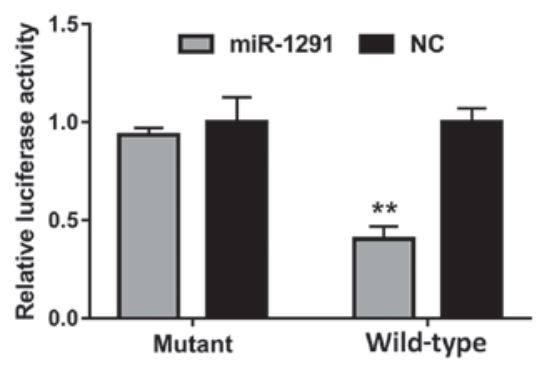

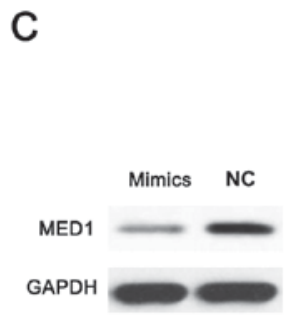

E

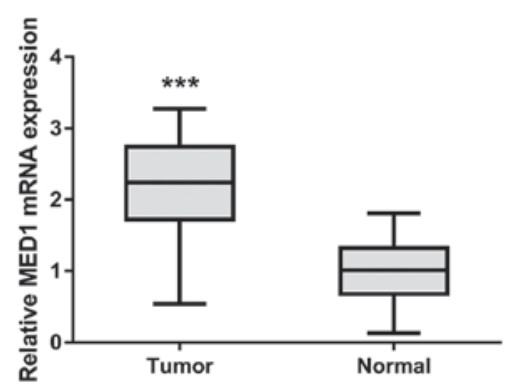

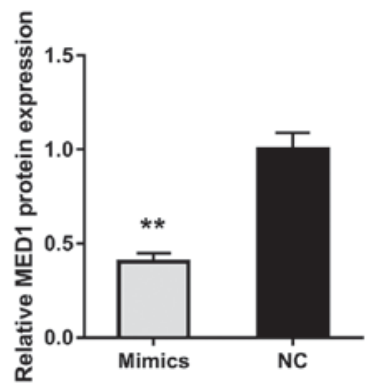
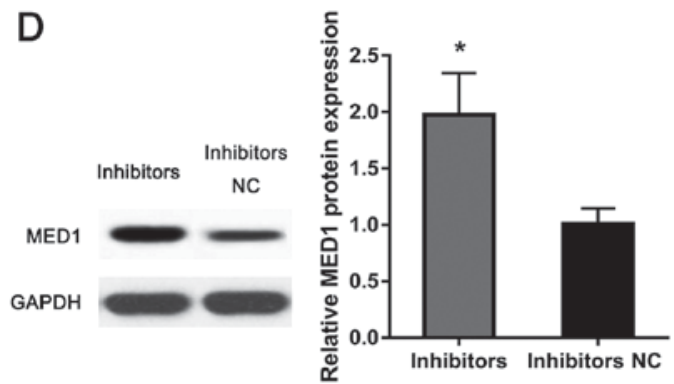

F

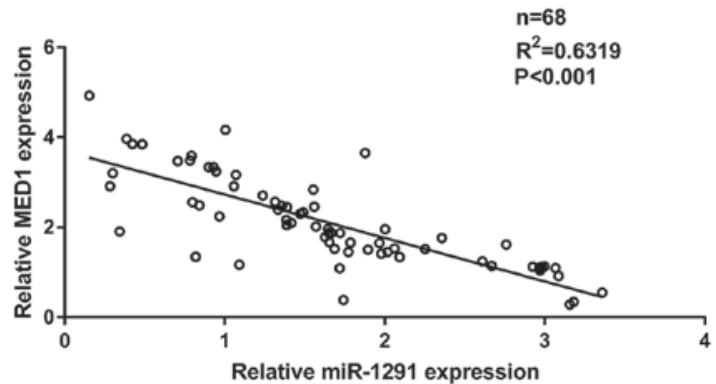

Figure 4. miR-1291 targets MED1 in PCa. (A) The predicted binding site of miR-1291 at the MED1 mRNA 3'-UTR. (B) A luciferase reporter assay using pMIR-REPORT (vector), pMIR-REPORT MED1-3'-UTR-WT, or MED1-3'-UTR-Mut plasmid in DU-145 cells transfected with the miR-1291 mimics or NC. (C and D) Western blot analysis of MED1 protein in established DU-145 and LNCaP cells. (E) The expression of MED1 mRNA in PCa specimens and paired adjacent normal tissues was analyzed by RT-qPCR. (F) Correlation between miR-1291 expression and the MED1 mRNA level in PCa specimens $\left(\mathrm{R}^{2}=0.6319\right.$, $\mathrm{P}<0.001) .{ }^{*} \mathrm{P}<0.05,{ }^{* *} \mathrm{P}<0.01$ and ${ }^{* * *} \mathrm{P}<0.001$. PCa, prostate cancer; 3'-UTR, 3'-untranslated region; NC, negative control.

3'-UTR of their target genes, we searched several databases including miRWalk (http://mirwalk.umm.uni-heidelberg.de/), TargetScan (http://www.targetscan.org), PicTar (https://pictar. mdc-berlin.de/) and miRanda (http://www.microrna.org/). After cross-checking, we found MED1 as a potential target for miR-1291. Using dual-luciferase assay, we employed pLG3 promoter vector containing wild-type and mutant 3'-UTR of MED1 to verify our hypothesis (Fig. 4A). The luciferase activity of the wild-type group showed a markedly decrease while the mutant group showed no difference compared to each relative control group (Fig. 4B). We also detected the MED1 protein level in established cells by western blot analysis and found that MED1 expression was lower in miR-1291 upregulated DU-145 cells while higher in miR-1291 downregulated LNCaP cells compared to the relative negative control group (Fig. 4C and D). Furthermore, the mRNA level of MED1 in 98 paired PCa tissues was measured and it was found to be significantly increased than that in the adjacent normal tissues (Fig. 4E). Moreover, we analyzed the relationship of miR-1291 and MED1 in PCa tissues and verified an obvious negative correlation between them $\left(R^{2}=0.6319\right.$, $\mathrm{P}<0.001)$. Our results suggest that MED1 as a direct target for miR-1291 in PCa.
Overexpression of MED1 restored the effect of miR-1291 upregulation. To further identify our assumption, we overexpressed MED1 in miR-1291 mimics-treated DU-145 cells. The MED1 protein level was obviously restored by pcDNA-MED1 (Fig. 5A). Using MTT assay, we found that the inhibition effect of miR-1291 on cell proliferation was restored by overexpression of MED1 (Fig. 5B). Also, upregulation of MED1 reversed the effect of miR-1291 on cell cycle (Fig. 5C). These results demonstrate that miR-1291 functions as a tumor suppressor in PCa via repressing MED1 expression.

miR-1291 inhibits tumor growth in vivo. To explore the influence of miR-1291 in PCa in vivo, we constructed a xenograft model using nude mice. As clearly shown in Fig. 6A, tumors grew significantly slower in miR-1291 mimics-treated group than the $\mathrm{NC}$ group. Also, we measured the weight of xenografts and found that the weight of miR-1291-upregulated group was obviously lower than that of the control group (Fig. 6B). Furthermore, the expression of miR-1291 and MED1 was detected using RT-qPCR. The MED1 expression was remarkably lower in miR-1291 mimics group compared with the NC group (Fig. 6C and D). Also, we detected MED1 protein 
A

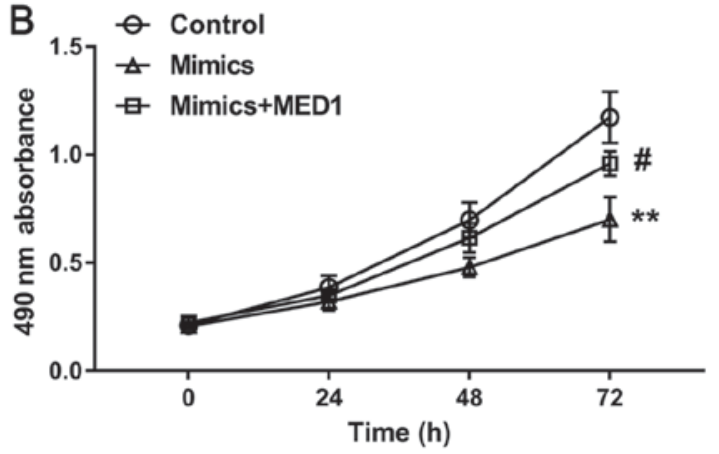

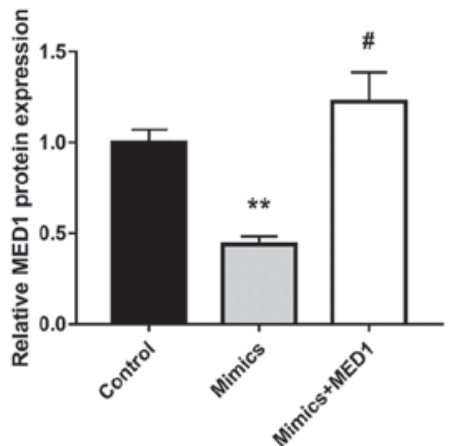

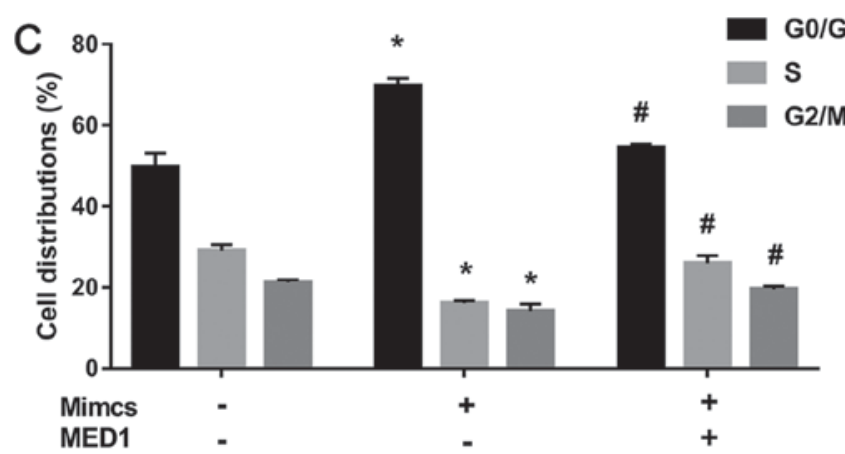

Figure 5. MED1 overexpression restores miR-1291-mediated inhibition of the growth of DU-145 cells. (A) Western blot analysis of MED1. GAPDH was used as an internal control. (B) Analysis of cell proliferation ability by MTT assay in control, mimics, or mimics + MED1 treated DU-145 cells. (C) The distributions of cell cycle were determined. Data are presented as mean $\pm \mathrm{SD}$ of three replicates. ${ }^{*} \mathrm{P}<0.05,{ }^{* *} \mathrm{P}<0.01$ and ${ }^{\#} \mathrm{P}<0.05$.

A

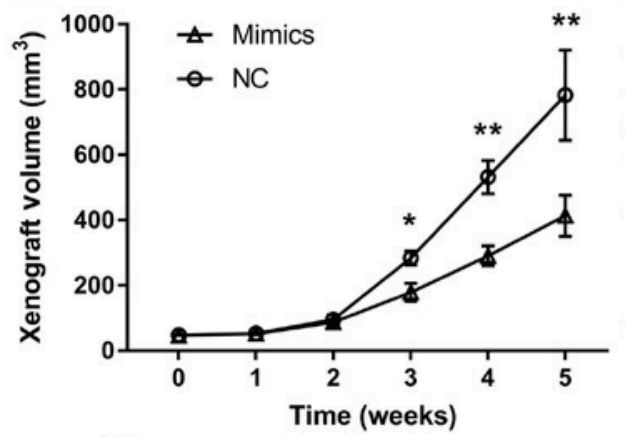

C

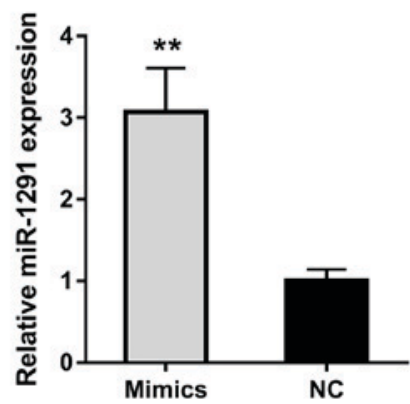

E

Mimics

MED1

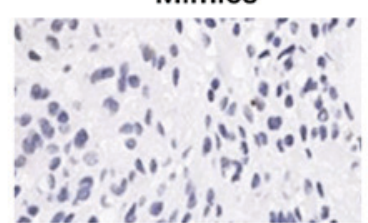

B

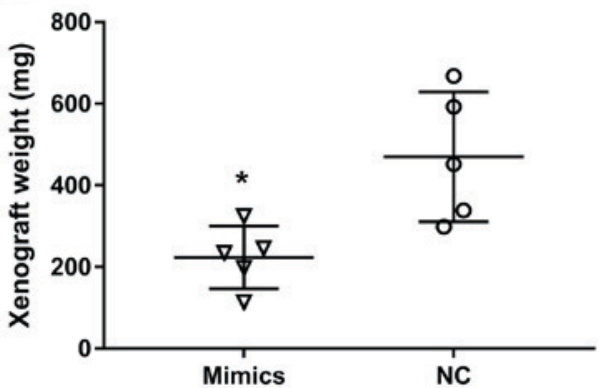

D

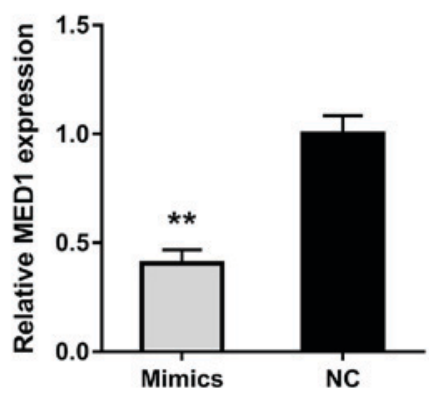

NC

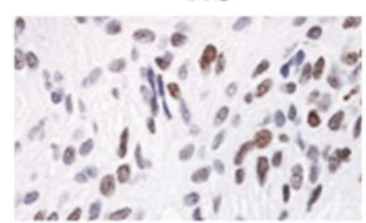

Figure 6. miR-1291 inhibits cell growth of PCa in vivo. (A) Tumor growth curves of xenografts. (B) The weight of xenografts was analyzed. (C) The expression of miR-1291 in xenografts was analyzed by RT-qPCR. (D) The expression of MED1 mRNA in xenografts was analyzed by RT-qPCR. (E) The expression of MED1 protein in xenografts was analyzed by IHC (magnification, $\mathrm{x} 400$ ). ${ }^{*} \mathrm{P}<0.05$ and ${ }^{* *} \mathrm{P}<0.01$. PCa, prostate cancer; IHC, immunohistochemistry. 
expression in xenografts using IHC and MED1 level decreased in the miR-1291 upregulation group (Fig. 6E). These results indicate that miR-1291 could inhibit $\mathrm{PCa}$ cell growth in vivo via downregulating MED1.

\section{Discussion}

With the increase of the incidence of $\mathrm{PCa}$, exploring the pathogenesis and progression of PCa has become an increasingly important research topic (3). In the present study we identified for the first time, up to our knowledge, that miR-1291 is significantly downregulated in PCa tissues and cells compared to normal tissues and cell lines. This result predicts that miR-1291 may serve as a new target in the pathogenesis and development of PCa.

As important factors regulating the progression of tumors, miRNAs have been reported to participate in the tumorigenesis and metastasis of PCa $(21,22)$. In the present study, we overexpressed and knocked down miR-1291 levels in PCa cell lines using miR-1291 mimics and inhibitors, and performed several functional experiments to confirm the role of miR-1291 in PCa. Upregulation of miR-1291 significantly inhibited PCa cell proliferation and cell cycle transition while downregulation of miR-1291 promoted cell growth. These assays indicate that miR-1291 could function as a tumor suppressor in $\mathrm{PCa}$. Furthermore, we verified MED1 as a direct target for miR-1291 in PCa via western blot analysis and luciferase assay. To our best knowledge, this is the first study to validate the relationship between miR-1291 and MED1 in PCa.

MED1 is a subunit of MED family which is a multiprotein complex and regulates eukaryotic mRNA synthesis (23). MED1 has been identified to play a role in the progression of several tumors. It acts as an antitumor gene in lung cancer and melanoma via inhibiting metastasis and invasion of cells $(23,24)$. In PCa, MED1 functions as a target of miR-205 and upregulation of MED1 has been associated with a poor prognosis of PCa (25). Furthermore, loss of MED1 obviously reduces proliferation of PCa cells $(26,27)$. In this study, we found that MED1 is inhibited by miR-1291 overexpression and its low expression causes the decrease of PCa cell growth, in consistency with the previously reported conclusions. Furthermore, we restored MED1 expression in miR-1291 overexpressed DU-145 cells and the ability of cell proliferation was restored. Taken together, we validated that miR-1291 inhibited PCa cell proliferation via repressing MED1.

We further constructed a xenograft model to verify the function of miR-1291 in PCa in vivo. With miR-1291 mimic treatment, tumors grew obviously slower than the control group and showed higher miR-1291 expression but lower MED1 level. Clearly, the protein level of MED1 in xenograft was repressed by $\mathrm{miR}-1291$. In combination with the results of previous in vitro experiments, we have reason to believe that miR-1291 could inhibit PCa tumorigenesis and progression through MED1. Although regulation of miR-1291 in $\mathrm{PCa}$ is likely a more complex network-like system, this study explains to a certain extent the role and mechanism of miR-1291 in PCa.

In conclusion, this study demonstrated that miR-1291 participates in the regulation of $\mathrm{PCa}$ progression and regulates cell proliferation by downregulating MED1 expression in vitro and in vivo. These findings might suggest miR-1291 as a novel target for PCa biological diagnosis and therapy.

\section{Acknowledgements}

Not applicable.

\section{Funding}

This study was supported by the National Natural Science Foundation of China (no.81602217), the Scienceand Technology Planning Project of Zhejiang Province (no. 2015C33096), and the Medical Scientific Research Foundation of Zhejiang Province (no. 2015117161).

\section{Availability of data and materials}

All data generated or analyzed during this study are included in this published article.

\section{Authors' contributions}

QC and WZ designed the study and performed the experiments. AZ, LR and JC acquired the data. KL and ZW analyzed the data. QC and WZ prepared the manuscript. All authors read and approved the final manuscript.

\section{Ethics approval and consent to participate}

The study was approved by the Ethics Committee of Tongde Hospital of Zhejiang Province (Hangzhou, China). Signed informed consents were obtained from the patients or the guardians.

\section{Patient consent for publication}

Not applicable.

\section{Competing interests}

The authors declare that they have no competing interests.

\section{References}

1. Siegel RL, Miller KD and Jemal A: Cancer statistics, 2018. CA Cancer J Clin 68: 7-30, 2018

2. Blumenthal-Barby JS, Lee D and Volk RJ: Toward ethically responsible choice architecture in prostate cancer treatment decision-making. CA Cancer J Clin 65: 257-260, 2015.

3. Chan JM, Stampfer MJ and Giovannucci EL: What causes prostate cancer? A brief summary of the epidemiology. Semin Cancer Biol 8: 263-273, 1998.

4. Levesque C and Nelson PS: Cellular constituents of the prostate stroma: Key contributors to prostate cancer progression and therapy resistance. Cold Spring Harb Perspect Med 8: pii: a030510, 2018.

5. Garisto JD and Klotz L: Active surveillance for prostate cancer: How to do it right. Oncology (Williston Park) 31: 333-340, 345, 2017.

6. Shepard DR and Raghavan D: Innovations in the systemic therapy of prostate cancer. Nat Rev Clin Oncol 7: 13-21, 2010.

7. Ameres SL and Zamore PD: Diversifying microRNA sequence and function. Nat Rev Mol Cell Biol 14: 475-488, 2013.

8. Berindan-Neagoe I, Monroig PC, Pasculli B and Calin GA: MicroRNAome genome: A treasure for cancer diagnosis and therapy. CA Cancer J Clin 64: 311-336, 2014. 
9. Carthew RW and Sontheimer EJ: Origins and mechanisms of miRNAs and siRNAs. Cell 136: 642-655, 2009.

10. Zhang B, Pan X, Cobb GP and Anderson TA: microRNAs as oncogenes and tumor suppressors. Dev Biol 302: 1-12, 2007.

11. Bartels CL and Tsongalis GJ: MicroRNAs: Novel biomarkers for human cancer. Clin Chem 55: 623-631, 2009.

12. Cai C, Chen QB, Han ZD, Zhang YQ, He HC, Chen JH, Chen YR, Yang SB, Wu YD, Zeng YR, et al: miR-195 inhibits tumor progression by targeting RPS6KB1 in human prostate cancer. Clin Cancer Res 21: 4922-4934, 2015.

13. Josson S, Gururajan M, Hu P, Shao C, Chu GY, Zhau HE, Liu C, Lao K, Lu CL, Lu YT, et al: miR-409-3p/-5p promotes tumorigenesis, epithelial-to-mesenchymal transition, and bone metastasis of human prostate cancer. Clin Cancer Res 20: 4636-4646, 2014.

14. Liu C, Kelnar K, Liu B, Chen X, Calhoun-Davis T, Li H, Patrawala L, Yan H, Jeter C, Honorio S, et al: The microRNA miR-34a inhibits prostate cancer stem cells and metastasis by directly repressing CD44. Nat Med 17: 211-215, 2011.

15. Rajendiran S, Parwani AV, Hare RJ, Dasgupta S, Roby RK and Vishwanatha JK: MicroRNA-940 suppresses prostate cancer migration and invasion by regulating MIEN1. Mol Cancer 13: $250,2014$.

16. Luo H, Guo W, Wang F, You Y, Wang J, Chen X, Wang J, Wang Y, Du Y, Chen X, et al: miR-1291 targets mucin 1 inhibiting cel proliferation and invasion to promote cell apoptosis in esophageal squamous cell carcinoma. Oncol Rep 34: 2665-2673, 2015.

17. Tu MJ, Pan YZ, Qiu JX, Kim EJ and Yu AM: MicroRNA-1291 targets the FOXA2-AGR2 pathway to suppress pancreatic cancer cell proliferation and tumorigenesis. Oncotarget 7: 45547-45561, 2016.

18. Yamasaki T, Seki N, Yoshino H, Itesako T, Yamada Y, Tatarano S, Hidaka $\mathrm{H}$, Yonezawa T, Nakagawa M and Enokida H: Tumor-suppressive microRNA-1291 directly regulates glucose transporter 1 in renal cell carcinoma. Cancer Sci 104: 1411-1419, 2013.

19. Soufi-Zomorrod M, Hajifathali A, Kouhkan F, Mehdizadeh M, Rad SM and Soleimani M: MicroRNAs modulating angiogenesis: miR-129-1 and miR-133 act as angio-miR in HUVECs. Tumour Biol 37: 9527-9534, 2016.
20. Livak KJ and Schmittgen TD: Analysis of relative gene expression data using real-time quantitative PCR and the 2(-Delta Delta C(T)) Method. Methods 25: 402-408, 2001.

21. Turkbey B, Brown AM, Sankineni S, Wood BJ, Pinto PA and Choyke PL: Multiparametric prostate magnetic resonance imaging in the evaluation of prostate cancer. CA Cancer J Clin 66: 326-336, 2016.

22. Fendler A, Stephan C, Yousef GM and Jung K: MicroRNAs as regulators of signal transduction in urological tumors. Clin Chem 57: 954-968, 2011.

23. Gade P, Singh AK, Roy SK, Reddy SP and Kalvakolanu DV: Down-regulation of the transcriptional mediator subunit Med1 contributes to the loss of expression of metastasis-associated dapk1 in human cancers and cancer cells. Int J Cancer 125: 1566-1574, 2009

24. Yang Y, Leonard M, Zhang Y, Zhao D, Mahmoud C, Khan S, Wang J, Lower EE and Zhang X: HER2-driven breast tumorigenesis relies upon interactions of the estrogen receptor with coactivator MED1. Cancer Res 78: 422-435, 2018.

25. Hulf T, Sibbritt T, Wiklund ED, Patterson K, Song JZ, Stirzaker C, Qu W, Nair S, Horvath LG, Armstrong NJ, et al: Epigenetic-induced repression of microRNA-205 is associated with MED1 activation and a poorer prognosis in localized prostate cancer. Oncogene 32: 2891-2899, 2013

26. Jin F, Irshad S, Yu W, Belakavadi M, Chekmareva M, Ittmann MM, Abate-Shen C and Fondell JD: ERK and AKT signaling drive MED1 overexpression in prostate cancer in association with elevated proliferation and tumorigenicity. Mol Cancer Res 11: 736-747, 2013

27. Chen Z, Zhang C, Wu D, Chen H, Rorick A, Zhang X and Wang Q: Phospho-MED1-enhanced UBE2C locus looping drives castration-resistant prostate cancer growth. EMBO J 30: 2405-2419, 2011

This work is licensed under a Creative Commons Attribution-NonCommercial-NoDerivatives 4.0 International (CC BY-NC-ND 4.0) License. 